\title{
Capon- and APES-Based SAR Processing: Performance and Practical Considerations
}

\author{
Paco López-Dekker, Member, IEEE, and Jordi J. Mallorquí, Member, IEEE
}

\begin{abstract}
This paper discusses the use of Capon's minimumvariance method (MVM) and Amplitude and Phase EStimation (APES) spectral-estimation algorithms to synthetic aperture radar range-azimuth focusing. The rationale of the algorithms is discussed. An implementation of a Capon or APES processing chain is explained, and processing parameters such as chip-image size, resampling factor, and diagonal loading are discussed. For multichannel cases, a joint-processing approach is presented. A set of Monte Carlo simulations are described and used to benchmark Capon- and APES-based processing against conventional matched-filter-based approaches. Both methods improve the resolution and reduce sidelobes. APES yields generally better estimates of amplitude and phase than Capon but with worse resolution. Results with RADARSAT-2 quad-polarization data over Barcelona are used to qualitatively study the real-life performance of these algorithms.
\end{abstract}

Index Terms-Adaptive processing, synthetic aperture radar, 2-D spectral analysis.

\section{INTRODUCTION}

$\mathbf{T}$ RADITIONALLY, most research in synthetic aperture radar (SAR) focusing algorithms has concentrated on the implementation of efficient and accurate approximations to a matched filter in range and azimuth, with some appropriate fixed tapering to mitigate range and azimuth sidelobes. In order to somehow separate the compression in range and azimuth, these algorithms need to deal with range cell migration and with the fact that the azimuth phase history is range dependent. Typical processing strategies include straightforward rangeDoppler algorithms, more refined wavenumber domain algorithms [1], and, more recently, chirp-scaling algorithms [2], [3]. The quality of these algorithms is usually evaluated by comparing them to exact time-domain backpropagation algorithms [4]. While efficient implementations of matched-filter-based SAR processors are still relevant in the context of batch processing of large amounts of SAR data, the ever-increasing available

Manuscript received March 27, 2009; revised August 19, 2009. First published February 17, 2010; current version published April 21, 2010. This work was supported in part by the Spanish Ministry of Science and Innovation (MICINN) and European Union FEDER funds under projects TEC200765690/TCM and TEC2008-06764-002-01, by the Spanish Ramon y Cajal Program, and by the Catalan Commission for Research (CIRIT).

P. López-Dekker was with the Remote Sensing Laboratory, Department of Signal Theory and Communications, Universitat Politècnica de Catalunya, 08034 Barcelona, Spain. He is now with the Microwaves and Radar Institute, German Aerospace Center (DLR), 82230 Oberpfaffenhofen, Germany (e-mail: Francisco.Dekker@dlr.de).

J. J. Mallorquí is with the Remote Sensing Laboratory, Department of Signal Theory and Communications, Universitat Politècnica de Catalunya, 08034 Barcelona, Spain (e-mail: mallorqui @tsc.upc.edu).

Color versions of one or more of the figures in this paper are available online at http://ieeexplore.iee.org.

Digital Object Identifier 10.1109/TGRS.2009.2038902 computing power should be taken as an invitation to explore alternative processing approaches.

Several authors have recognized that SAR focusing can be cast as a spectral-estimation problem and that, therefore, a wide range of spectral-estimation methods can be applied to it [5]. Of these methods, nonparametric adaptive methods, such as Capon's minimum-variance method (MVM) [6] and Amplitude and Phase EStimation (APES), seem to be some of the most promising approaches [5], [7]-[9].

It is worth noting that these types of advanced highresolution spectral-estimation methods are routinely applied to problems closely related to SAR focusing. For example, Capon's MVM is one of the preferred digital beamforming algorithms applied in vertically pointed mesospheretroposphere-stratosphere (MST) imaging radars [10]. It is also used as a nonparametric beamformer in SAR tomography [11]. A general overview and discussion of nonparametric and parametric spectral estimators, such as MUSIC, and their application to array processing can be found in [12].

This paper studies the application of Capon and APES to SAR focusing. Given the use of Capon's method in related problems, it is only natural to thoroughly explore its application to this one. Like many other spectral estimators, Capon requires an estimate of the covariance matrix that statistically describes the data. In many applications, the covariance matrix in some dimension can be estimated by averaging over an independent dimension. For example, in MST radars, ergodicity is assumed to robustly estimate the spatial covariance matrix by replacing the expected values with time averages. In SAR tomography, if the scene is assumed to be homogeneous, the covariance matrix in the tomographic dimension can be obtained by averaging in range and azimuth. For 2-D SAR focusing, however, there is no free dimension left, over which averages are computed. Instead, averages are calculated over sets of overlapping sublooks. This endogenous use of the data can easily lead to overadaptation, causing artifacts in the resulting data. APES is closely related to Capon but, by construction, less prone to introducing artifacts related to overadaptation.

While other authors have focused primarily on the signal processing side of the problem, treating SAR focusing primarily as an example, this paper studies the practical aspects and caveats of SAR focusing using these algorithms.

Section II reformulates the SAR focusing problem as a 2-D spectral-estimation problem and discusses the benefits of using the Capon and APES adaptive estimators. Section III discusses the implementation of a Capon/APES-based SAR processing chain. The Capon and APES estimators are basically treated as black boxes, although some details regarding the efficient implementation used in this paper are provided in the Appendix. Several processing parameters are discussed, and the option 
of jointly processing the available channels in a multichannel context is considered. In Section IV, the performance of Capon and APES is benchmarked by numerically analyzing the results of Monte Carlo simulations. Section V shows and discusses the results using real RADARSAT-2 data, while Section VI offers concluding remarks.

\section{Formulation}

\section{A. Spectral Estimation in SAR Processing}

Let us consider the point-target response of a reference target, $p_{0}\left(t_{r}, t_{a}\right)$, where $t_{r}$ represents the range delay (or fast time) and $t_{a}$ is the azimuth delay (or slow time). The received signal for a target in the vicinity of this reference target is

$$
z\left(t_{r}, t_{a}\right)=a_{1} \cdot p_{0}\left(t_{r}-t_{r_{1}}, t_{a}-t_{a_{1}}\right)
$$

which is a slow- and fast-time-delayed replica of the reference point-target response, with $t_{r_{1}}$ and $t_{a_{1}}$ indicating the range and azimuth temporal delays of the target with respect to the reference target, respectively. A matched-filter approach to process this received signal is to calculate its convolution with the time-reversed conjugate of the point-target response

$$
s\left(t_{r}, t_{a}\right)=z\left(t_{r}, t_{a}\right) * p_{0}^{*}\left(-t_{r},-t_{a}\right) .
$$

In the frequency domain, the processed image is given by

$$
S\left(f_{r}, f_{a}\right)=Z\left(f_{r}, f_{a}\right) \cdot P_{0}^{*}\left(f_{r}, f_{a}\right)
$$

where $Z\left(f_{r}, f_{a}\right)$ and $P_{0}\left(f_{r}, f_{a}\right)$ are the 2-D Fourier transforms of the received signal and the point-target response, respectively. Because the received signal is a time-delayed replica of $p_{0}\left(t_{r}, t_{a}\right)$, its Fourier transform can be expressed as

$$
Z\left(f_{r}, f_{a}\right)=a_{1} \cdot P_{0}\left(f_{r}, f_{a}\right) \cdot e^{-j 2 \pi\left(t_{r_{1}} \cdot f_{r}+t_{a_{1}} \cdot f_{a}\right)}
$$

and, therefore

$$
S\left(f_{r}, f_{a}\right)=\left|P_{0}\left(f_{r}, f_{a}\right)\right|^{2} \cdot a_{1} \cdot e^{-j 2 \pi\left(t_{r_{1}} \cdot f_{r}+t_{a_{1}} \cdot f_{a}\right)} .
$$

This resulting expression is that of a 2-D complex harmonic function in the frequency domain windowed by the spectral density function of the point-target response. Thus, the step of taking the inverse Fourier transform of (3) to obtain (2) can be considered as the simplest implementation of a spectral estimator, which may be replaceable by more sophisticated ones.

In this paper, the use of Capon's MVM and APES spectral estimators is considered. As a simple preliminary example, the upper left panel of Fig. 1 shows images of five randomly placed targets focused using a straight discrete Fourier transform (DFT; strict matched filter), a DFT after 2-D Hamming tapering, the APES estimator, and the Capon estimator (with some amount of diagonal loading, which will be discussed later), respectively. In all cases, the input data were critically sampled at the Nyquist rate. The focused images were generated with a range and azimuth sampling rate that was a factor of eight higher than the nominal resolution given by the available bandwidth. The APES and Capon estimators both reduce the target sidelobes while also improving the resolution of the targets. In this example, the phases of the targets estimated using a DFT, a windowed DFT, or APES were all very close to the nominal phases. In contrast, Capon resulted in phase errors over $5^{\circ}$. The upper right panel shows the same comparison for a case in which a uniform clutter scene was simulated by placing a large number of random targets per resolution cell. Qualitatively, it is interesting to observe that the APES and Capon estimators produce results that are similar to the Hamming-Fourier case.

\section{B. Capon and APES Rationale}

It is important to understand the limitations of these estimators in order to use them in SAR processing. It is not in the scope of this paper to discuss the theoretical and mathematical details of neither the Capon nor the APES estimator. Instead, the objective of this discussion is to provide insight on what these algorithms do and their implications.

Both algorithms construct a bank of adaptive bandpass finiteimpulse-response filters of length $M$. Considering, for simplicity, the 1-D case, the output of each of these filters is

$$
\alpha_{\omega}[l]=h_{\omega}[l] * z[l]=\mathbf{h}_{\omega}^{H} \mathbf{z}_{l}
$$

where $h_{\omega}[l]$ represents the impulse response for the filter with center frequency $\omega$ and

$$
\mathbf{z}_{l}=\left[\begin{array}{llll}
z[l] & z[l+1] & \cdots & z[l+M-1]
\end{array}\right]^{\mathrm{T}}
$$

is the vector of data samples.

In Capon's case, these filters are designed so that the power of the filtered signal is minimized with the constraint that, in the frequency domain, the filter must have unity gain at the selected frequency [6]. In a 1-D case, for a data set with covariance matrix $\mathbf{R}$, the optimal filter of length $M(\mathbf{h})$ for frequency $\omega$ is given by the solution to

$$
\min _{\mathbf{h}} \mathbf{h}^{H} \mathbf{R h}, \quad \text { subject to } \quad \mathbf{h}^{H} \mathbf{a}_{\mathbf{M}}=1
$$

with

$$
\mathbf{a}_{\mathbf{M}}=\left[\begin{array}{lllll}
1 & e^{j \omega} & e^{j 2 \omega} & \cdots & e^{j(M-1) \omega}
\end{array}\right]^{\mathrm{T}}
$$

In the presence of a small number of dominant frequency components (which translates to a small number of bright targets), the filters used to estimate spectral components will tend to place nulls at those dominant frequencies. In contrast, the filters are allowed to have a large frequency response (or sidelobes) at those frequencies at which there is less interfering signal. The apparent resolution increases because of the ability of the algorithm to place a null-suppressing spectral spillover from a nearby object. Of course, as the number of dominant spectral components that need to be suppressed increases (in relation to the number of data samples available), the capacity of the algorithm to suppress them decreases. In the limit of uniform clutter, the algorithm cannot perform better than an optimum nonadaptive filter, as shown in Fig. 1.

Several authors have found that the power-minimizing criterion applied in the derivation of the Capon estimator results in a tendency to underestimate the spectral power. As a proposed solution, the APES estimator minimizes the mean-square difference between the filter output and the (estimated) harmonic 

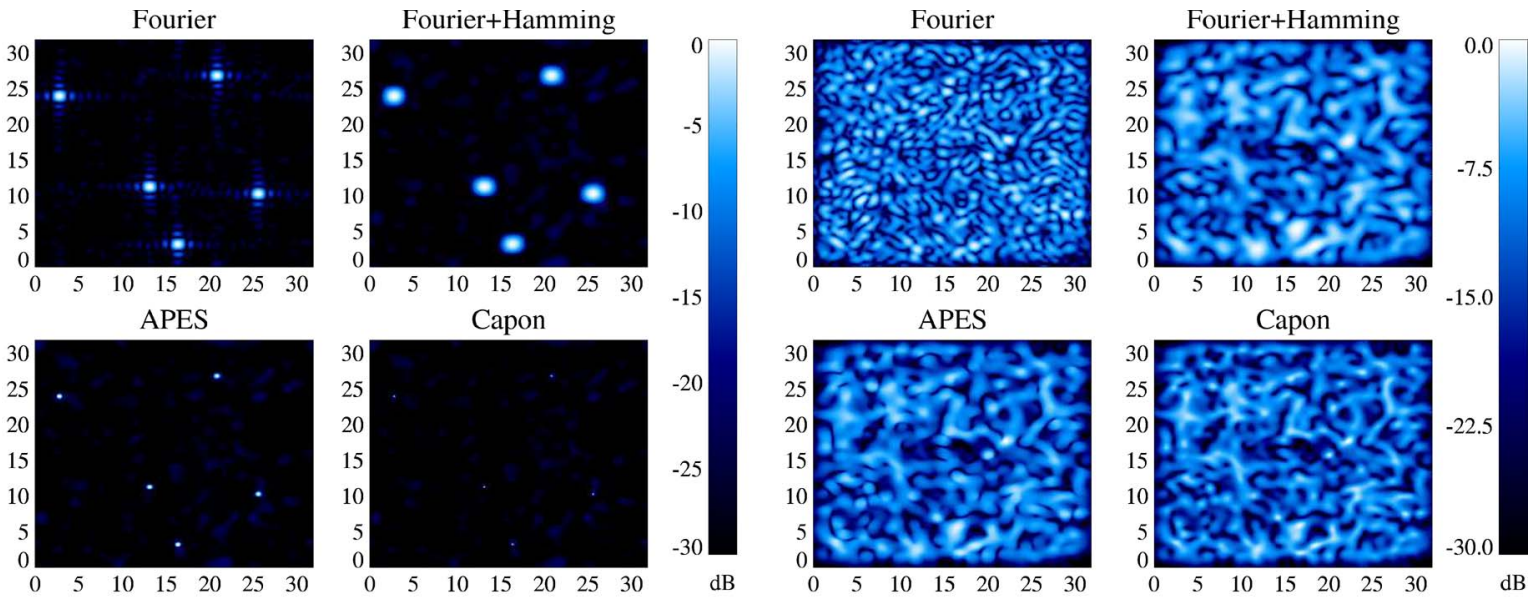

(a)
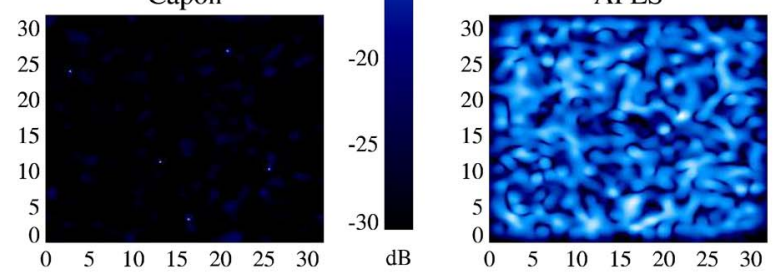

(b)
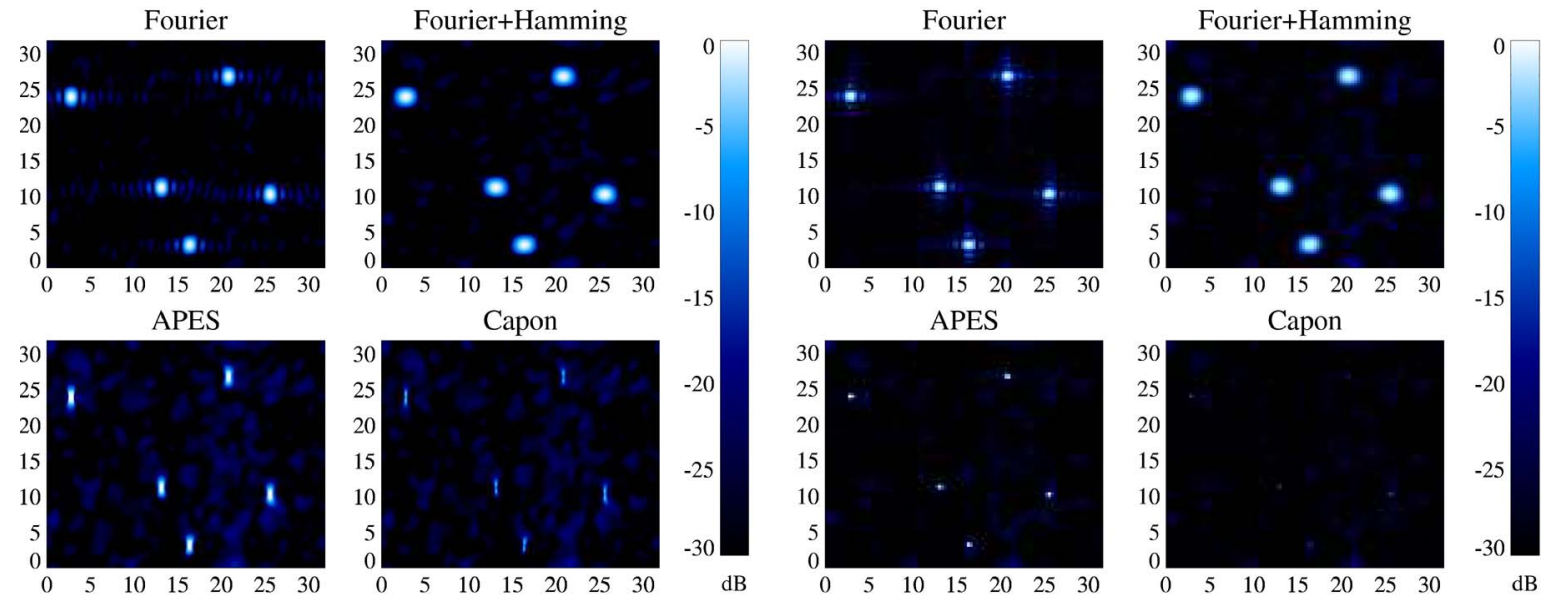

(c)
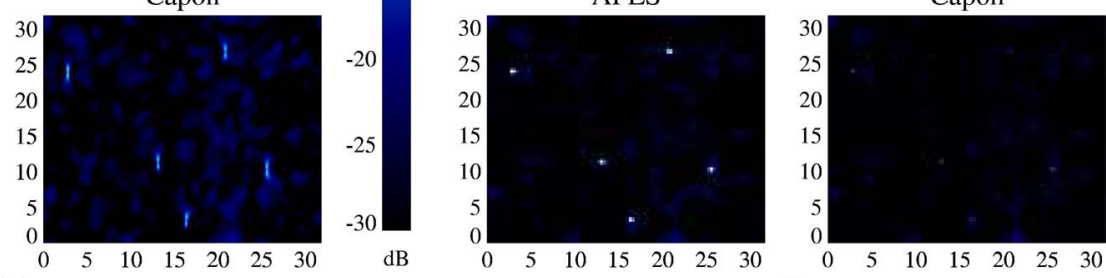

(d)

Fig. 1. Upper panels: (Left) Five-target scene and (right) clutter scene processed using a DFT without windowing, a DFT after applying a 2-D Hamming window, and the APES and Capon estimators. Lower panels: (Left) Impact of beam-pattern tapering on the azimuth resolution and (right) impact of low resampling. (a) Five targets. (b) Clutter. (c) Azimuth tapering. (d) Low resampling.

component at each frequency [7]. Again, for a 1-D case, this can be expressed as

$$
\min _{\mathbf{h}, \alpha} \overline{\left|\mathbf{h}^{H} \mathbf{z}_{\mathbf{l}}-\alpha e^{j \omega l}\right|^{2}}, \quad \text { subject to } \quad \mathbf{h}^{H} \mathbf{a}_{\mathbf{M}}=1 .
$$

In this case, a high-power signal is allowed at the output of each adaptive filter as long as it looks like a clean sinusoidal or complex exponential function. These authors find that the APES estimator shows a lower bias at the cost of a lower resolution and a significantly higher computational cost.

Both algorithms require knowledge of the covariance matrix of the data and computation of its inverse. In practice, this covariance matrix can only be estimated from the data. Errors in this estimate may result in the appearance of artifacts in the final image.

An important consideration is that these algorithms are conceived to estimate the amplitude of harmonic functions, i.e., sinusoidal or complex exponential functions with constant amplitude. Thus, any amplitude tapering of the data will harm the performance of the algorithm. In the case of SAR processing, this is important because the beam pattern tapers the data in the azimuth dimension. To illustrate this, the previous five-target simulation has been modified by applying a Hamming window to the data in the azimuth direction [see Fig. 1(c)]. As expected, its effect in the Fourier case is to reduce the azimuth sidelobes and resolution. In the Fourier-Hamming case, only additional range tapering was introduced, so the result is virtually identical to that shown in Fig. 1. However, for APES- or Capon-based processing, this beam-pattern tapering results in a dramatic loss of azimuth resolution.

\section{Resolution}

As discussed in the Introduction, the term "resolution" is a vague and sometimes misleading concept. Nevertheless, for nonadaptive matched-filter-based methods, the resolution can be unambiguously defined as the $3-\mathrm{dB}$ width of the point-target response. In contrast, using an adaptive estimator, this 3-dB width may vary for each particular target, depending on the nature of the scene. Bright isolated targets will be much better resolved than weak targets in a cluttered portion of the image. This variability of the final resolution and the associated uncertainty are, by themselves, a negative thing because they complicate the qualitative and quantitative interpretations of the resulting image and because they may hinder their statistical analysis. However, in many cases, SAR images are rather sparse, which allows a significant improvement of the image 
resolution, even though it is hard to establish by how much. A theoretical discussion of the probability of resolution for two targets for MVM and APES can be found in [13] and [14], respectively. For the Capon estimator, it is found that the minimum distance at which two targets are resolved can be arbitrarily reduced (improved) by increasing the signal-tonoise ratio (SNR). For APES, however, this resolution tends asymptotically to a finite limit. The main benefit of APES with respect to a conventional matched filter is its capability to resolve targets of unequal power.

There is a practical consequence of this varying resolution. The grid spacing of the generated image should be fine enough to capture the high-resolution features produced by adaptive focusing. For example, Fig. 1(c) shows the same image as Fig. 1(a) but with a grid spacing that is only a factor of three higher than the nominal resolution in both dimensions. In Capon's case, four out of the five targets seem to have lost most of their intensity but only because the narrow peaks corresponding to these targets are not being sampled properly.

\section{IMPLEMENTATION}

\section{A. Processing Flow}

This section discusses the implementation of a SAR processor based on the Capon or APES spectral estimators. It extends the processing flow described in [5]. In implementing a Caponor APES-based SAR processor, the following aspects should be considered.

1) These algorithms are CPU and memory intensive and can only be applied to SAR processing if the image is divided into small chip images.

2) Following Amdahl's argument [15], it can be argued that, given the computational cost of these spectral estimators, an optimization of the rest of the processing flow should have a small impact on the overall processing time.

Fig. 2 shows a flowchart of the proposed processing chain, while some of the steps are further shown in Fig. 3. The input is a raw data set or a stack of data sets that could correspond to interferometric or polarimetric channels. The processing chain performs the following steps.

1) Standard $S A R$ processing: First, the data are focused using any conventional SAR algorithm. In principle, this step can be stepped over if focused single-look complex data are provided. If this focusing step is included, no azimuth or range windowing should be applied. Note also that if a stack of images is considered, these should also be coregistered.

2) Spectral equalization: In this step, the 2-D power spectral density is equalized to compensate the beam-pattern weighting and any azimuth or range windowing previously introduced, and eliminate the rippling of the power spectra associated to the chirp waveform. This equalizer is derived from the nominal chirp parameters, azimuth phase history, and azimuth antenna pattern. The signal is also band limited to avoid an excessive amplification of the noise spectrum. Also, band limiting will, in azimuth, limit the impact of ambiguous targets, which could otherwise be increased by equalization. After this equalization, the power spectrum of the image should be essentially a 2-D rectangular function.

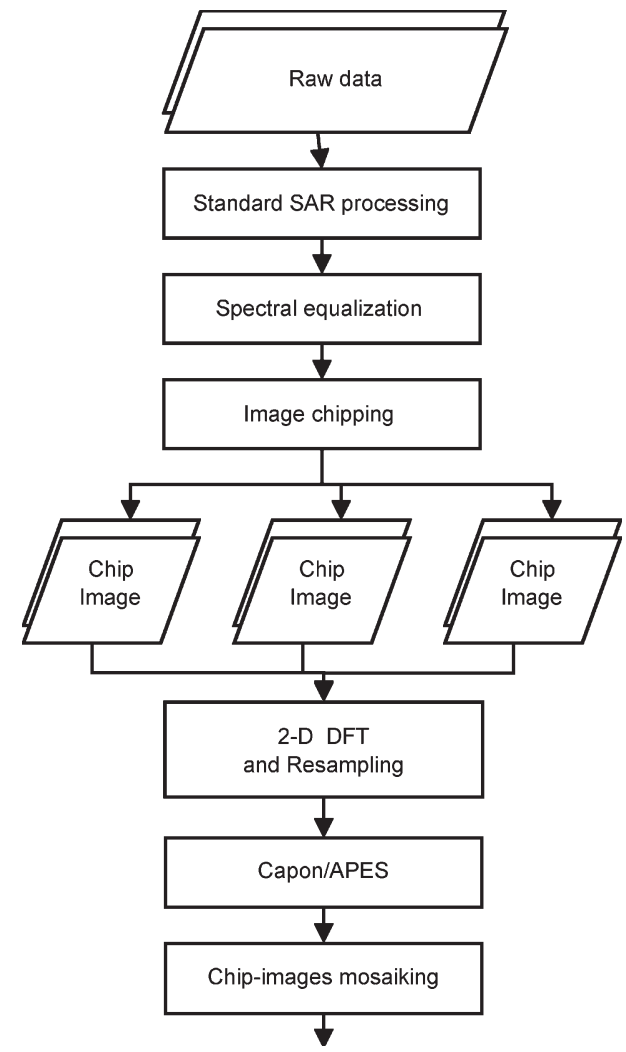

Fig. 2. Flowchart of the proposed Capon/APES-based processing chain.

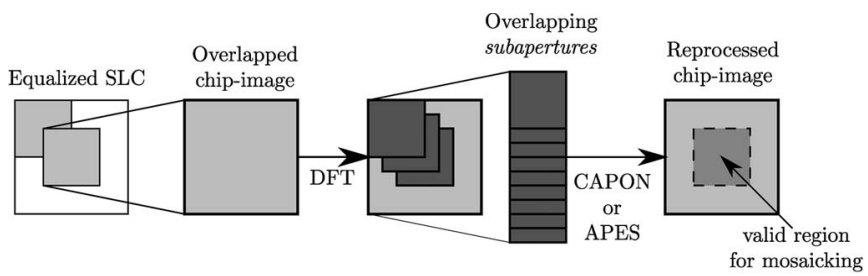

Fig. 3. Illustration of the division of the equalized SLC image into a set of overlapped chip images. After applying a DFT, the frequency-domain chip images are arranged in an ensemble of overlapping subapertures, which is used to estimate the covariance matrix used by Capon and APES.

3) Image chipping: The equalized image is divided into a set of overlapping small images or stacks of interferometric or polarimetric chip images. This is shown in Fig. 3 for the single-channel case. The size of these images depends primarily on the available computing resources. Overlapping is necessary to avoid edge effects. Like what other authors did, in the results presented in this paper, a $50 \%$ overlap has been applied in both dimensions, which implies that the processing burden is increased by a factor of four.

4) Two-dimensional DFT and resampling: Each chip image is brought back to the frequency domain, and the resulting 2-D data sets are critically resampled by dropping the lines and columns corresponding to frequencies filtered out during the previous equalization. It is compulsory that the data are shifted to baseband, as it would be done in a zero-padding interpolation process.

5) Capon/APES: The Capon or APES algorithm is applied to each chip image (or stack of images), thus obtaining 
a new set of adaptively focused and interpolated chip images.

6) Mosaicking: The last step consists of combining the center part of each chip image into a single image.

\section{B. Processing Parameters}

Although we purposely treat the Capon and APES algorithms as black boxes, it is necessary to discuss some aspects of these algorithms that can be tuned. More details can be found in the Appendix. The input of the estimator is a stack of $K N_{1} \times N_{2}$ frequency-domain chip images, where $K$ is the number of interferometric or polarimetric channels being simultaneously processed. Both algorithms require the estimation of the covariance matrix $(\mathbf{R})$ that describes these chip images. In order to obtain this estimate $(\hat{\mathbf{R}})$, the frequencydomain chip images are divided into an ensemble of $L_{1} \times L_{2}$ overlapping subapertures of $M_{1} \times M_{2}$ elements each. This is shown in Fig. 3. Different authors have found that maximal overlap provides the best results, so that $N_{1}=M_{1}+L_{1}-1$ and $N_{2}=M_{2}+L_{2}-1$. The covariance matrix describing an $M_{1} \times M_{2}$ data set is an $\left(M_{1} M_{2}\right) \times\left(M_{1} M_{2}\right)$ matrix, which cannot be singular. Assuming that the $K L_{1} L_{2}$ overlapping chip images are linearly independent and forward-backward averaging [5], it is necessary to satisfy

$$
M_{1} M_{2} \leq 2 K L_{1} L_{2}
$$

Taking

$$
M_{1}=\eta N_{1} \quad M_{2}=\eta N_{2}
$$

this yields the (overconstrained) upper bound

$$
\eta \lesssim \frac{\sqrt{2 K}}{\sqrt{2 K}+1} .
$$

Reducing this factor $\eta$ increases the number of overlapping images in the ensemble and therefore improves the quality of the covariance matrix estimate. This is important in order to avoid the appearance of artifacts in the result. However, taking smaller images causes a loss of resolution in the output image. APES is significantly more robust than Capon to the appearance of errors associated to low ensemble averaging in the estimation of $\hat{\mathbf{R}}$ [16]. In practice, a subaperture factor $\eta=0.5$ seems to be a good compromise between stability and resolution.

Resampling Factor: A critical parameter is the resampling factor. As shown in Fig. 1, Capon and APES can resolve singular targets with a significantly finer resolution than the nominal resolution of the system. Therefore, in order to guarantee that all targets are recovered, it is necessary to generate the output image with an appropriately higher sampling rate. A nontrivial issue is that the necessary resampling factor depends on the nature of the imaged scene as follows.

1) A better SNR improves the resolution and requires a higher resampling factor.

2) As the scene becomes more distributed, the resolution gain decreases, as well as the required resampling factor.

Thus, as the covariance matrix approaches a diagonal matrix, the resolution of both algorithms is degraded. In the limit, if the received signal corresponds to thermal noise or to a uniform background, in which case the covariance matrix becomes a diagonal matrix, the resolution is set by the bandwidth of the overlapping frequency-domain chip images used to estimate the covariance matrix. Therefore, in this case, the resolution is degraded by factor $\eta$, implicitly defined in (12), with respect to the nominal resolution.

This discussion also suggests that the resolution of the generated images can be limited by diagonally loading the estimated covariance matrix, which will be discussed next.

Diagonal Loading: Several authors have found (e.g., see [17]-[19]) that the stability of the estimation of the covariance matrix can be improved by diagonally loading this estimate

$$
\hat{\mathbf{R}}_{\gamma}=\hat{\mathbf{R}}+\gamma \mathbf{I}
$$

This is, to some extent, equivalent to increasing the white-noise content of the data. Diagonal loading robustifies the process by conditioning $\hat{\mathbf{R}}$. If $\gamma$ is chosen above the white-noise level of the data, it will prevent the estimators from adapting to a realization of white noise, thus avoiding the appearance of weak artifacts. If only weak targets and noise are present, then $\hat{\mathbf{R}}_{\gamma}$ will tend to $\gamma \mathbf{I}$ and the Capon estimator will tend to a straight DFT. Strong targets whose signals dominate $\hat{\mathbf{R}}$ will similarly dominate $\hat{\mathbf{R}}_{\gamma}$.

In our implementation of Capon and APES, parameter $\gamma$ is calculated by setting an equivalent $S N R_{\mathrm{dl}}$. For each data block, the received signal power is estimated by averaging the diagonal elements of the covariance matrix, and $\gamma$ is obtained by dividing this power by $S N R_{\mathrm{dl}}$. This yields

$$
\gamma=\frac{\operatorname{tr}(\hat{\mathbf{R}})}{S N R_{\mathrm{dl}} M_{1} M_{2}}
$$

where $\operatorname{tr}(\cdot)$ indicates the trace operator.

\section{Multichannel Processing: Joint Versus Independent Processing}

In many cases, there are multiple SAR images of the same scene. These images may correspond, for example, to single- or repeat-pass interferometric data sets, or to multiple polarimetric channels in PolSAR, or a combination of both in PolInSAR. With conventional matched-filter-based SAR processing, these channels are processed independently. For adaptive processing, it makes sense to also consider jointly processing all or a subset of the available channels.

For Capon, this joint processing has been implemented simply by using all channels to estimate the required covariance matrix. This assumes implicitly that the different channels are observing the same set of scattering centers and that differences are caused by speckle noise (fading). In that case, the channelaveraged covariance matrix should be a better estimate of the true one, which should improve the end result.

For APES, we have followed the formulation in [16], extending it from the two-channel interferometric case to a general multichannel case. The algorithm has been implemented by extending the efficient implementation of APES proposed in [9] to this multichannel case. Both extensions are discussed in detail in the Appendix. 


\section{Simulations AND Benchmarking}

\section{A. Simulation Procedure}

The adaptive nature of Capon/APES-based processing implies that the standard analysis of the point-target response is not significant. Instead, the analysis of the algorithm needs to be done in complex scenarios that include multiple targets. In order to evaluate the performance of Capon- and APES-based SAR focusing in a controlled environment, a large number of numerical simulations have been done. For these numerical tests, $32 \times 32$ sample interferometric pairs of chip images were generated with a varying number of simulated point targets. The interferometric pairs were identical, except that the phase of the targets had random $15^{\circ}$ root-mean-square (rms) difference. The simulation factors that were varied were as follows.

1) SNR: Random white noise was added to each chip image by changing the average single-target unprocessed $S N R_{s}$ between 0 and $20 \mathrm{~dB}$. Noise was assumed uncorrelated between interferometric pairs. Note that because the chip images are partially compressed images, these SNR values correspond to much lower raw-data single-target SNR values. For example, considering a total processing gain of $60 \mathrm{~dB}$ (which is in the ballpark of a typical orbital SAR mission), $30 \mathrm{~dB}\left(10 \log _{10} 32^{2}\right)$ of this gain corresponds to the nominal chip-image processing gain, and the remaining $30 \mathrm{~dB}$ would correspond to the partial compression. Therefore, in this particular example, the single-target raw-data SNR would be $30 \mathrm{~dB}$ lower, which is between -30 and $-10 \mathrm{~dB}$.

2) Target density: The number of targets in each chip image was varied between 4 and 512, which corresponds to a target density between 0.0039 and 0.5 targets per nominalresolution cell.

3) Diagonal loading: Each set of images was processed without any diagonal loading and by applying a diagonal loading corresponding to equivalent SNRs ranging from 0 to $20 \mathrm{~dB}$.

4) Resampling factor: The images were analyzed by applying resampling factors (I's) of four, six, and eight. For a matched filter, this resampling factor is an ideal interpolation factor.

5) Targets on grid: To evaluate the intrinsic performance of the algorithms and force that no targets were being misssampled, the target positions were optionally rounded to the nearest output grid point.

In order to gain statistical significance, for each combination of parameters, 20 realizations were simulated.

For the Capon and APES estimators, $\eta$ was set fixed to 0.5 . The chip images were also processed by applying a 2-D DFT, which corresponds to processing the original raw data using an unwindowed matched filter, and applying a 2-D DFT after applying a 2-D Hamming window for sidelobe suppression.

\section{B. Benchmarking}

In order to compare the performance of the proposed methods, we suggest a number of metrics. Considering first the amplitude of the images, the following four quantities and procedures have been used.

1) Amplitude bias: An amplitude bias is evaluated by dividing the amplitude of the processed images at the pixels corresponding to the nominal position of the targets (rounded to the nearest grid-point) by the amplitude of those targets, and averaging over all the targets and realizations. Targets spaced from another target less than the nominal resolution of the system are not considered.

2) Integrated-to-nominal-power ratio: To evaluate the resolution of the generated images, the total energy of each image is calculated and divided by the nominal energy, where this nominal energy is obtained assuming that each target contributes with a constant power in a I $\times$ I pixel region. Assuming that most of the energy is concentrated in the mainlobes, the Integrated to Nominal Power Ratio (INPR)

$$
\mathrm{INPR}=\frac{\sum_{i, j} P_{i, j}}{I^{2} \sum_{k} P_{t, k}}
$$

should tend to 1 for a matched filter, be greater than 1 if the resolution is degraded and be smaller than 1 if the resolution is improved. Note that an amplitude bias should also bias INPR. For an unbiased image, INPR is lower bounded by $1 / I^{2}$.

3) Average-sidelobe ratio (ASLR): An ASLR is calculated by evaluating the average power outside the nominal main lobes of all targets and dividing by the average target intensity. Thus, to calculate ASLR, first, rectangular regions centered around the nominal position of each target are masked out and the average power is calculated over the remaining pixels. For the results shown, the size of this target mask was set to $2 I \times 2 I$ pixels for the matched filter and $4 I \times 4 I$ for the Hamming-windowed case in order to accommodate the larger main lobe. This larger size mask was also applied for Capon and APES, for which the main-lobe size is data dependent, to accommodate the worst cases. Note that this procedure does not make sense for very high target densities for which the entire image would be masked out.

4) Peak-sidelobe ratio: A PSLR is calculated following the same procedure as that for an ASLR but taking the peak power of the pixels that have not been masked out. In the results presented, the PSLR was calculated for each realization and averaged over the set of realizations. It is worth noting that this definition of PSLR differs from the classical definition of PSLR in that the sidelobe intensity is compared with the average main-lobe intensity. Both definitions are compatible in the case of a single target.

The interferometric phase quality has been evaluated by simply calculating its rms error. Only points corresponding to the nominal position of a target have been considered, and like in the case of amplitude bias, targets that were within the nominal-resolution distance from another target were excluded. 

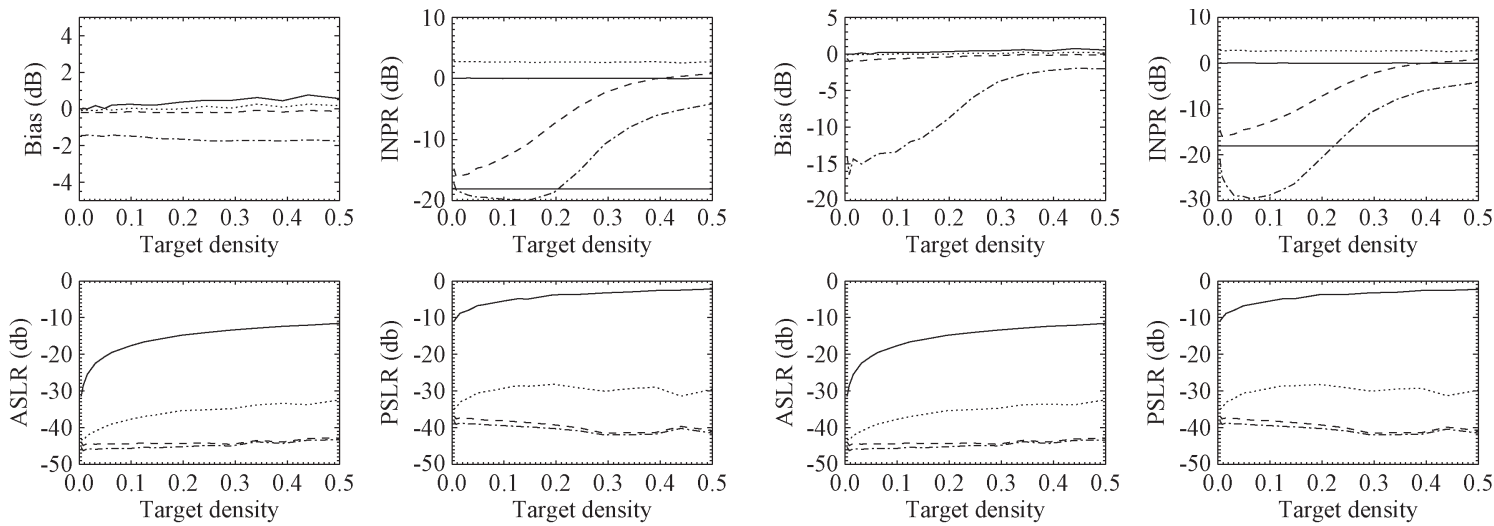

(a)
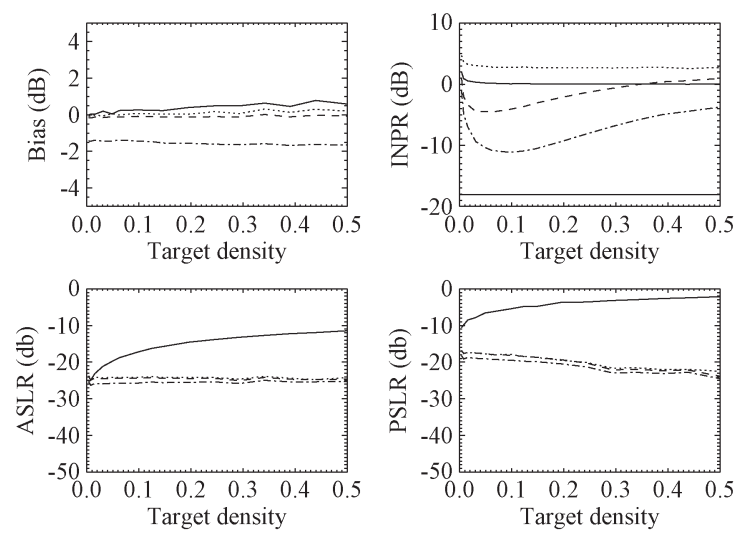

(c)
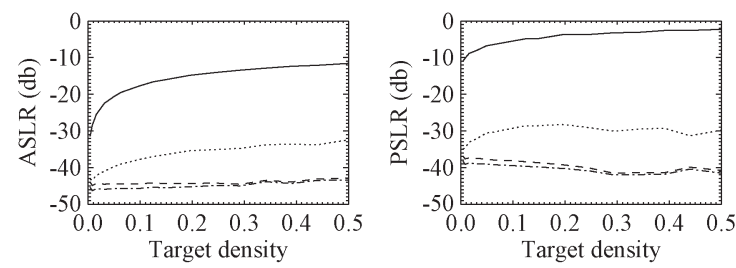

(b)
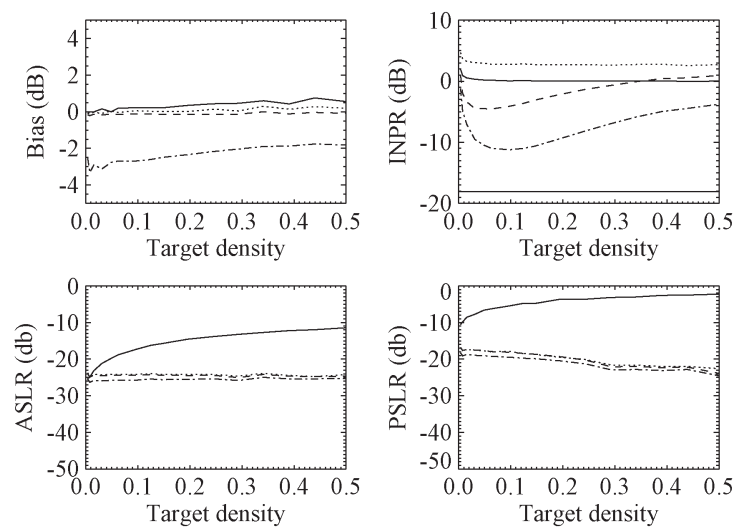

(d)

Fig. 4. Amplitude bias, INPR, ASLR, and PSLR as functions of target density. In all plots, the solid line corresponds to DFT processing (matched filter), the dotted line to DFT after applying a 2-D Hamming window, the dashed line to the APES estimator, and the dash-dot line to Capon processing. As a reference, the INPR plot shows also the $1 / I^{2}$ nominal lower bound. These results correspond to simulations, setting the resampling factor $I=8$, random amplitude, and $S N R_{s}=17 \mathrm{~dB}$ for the upper panels and $S N R_{s}=-3 \mathrm{~dB}$ for the lower ones. For the left panels, the targets were forced to lay on a grid point of the output image. (a) Targets on grid $\left(S N R_{s}=17 \mathrm{~dB}\right)$. (b) Targets off grid $\left(S N R_{s}=17 \mathrm{~dB}\right)$. (c) Targets on grid $\left(S N R_{s}=-3 \mathrm{~dB}\right)$. (d) Targets off grid $\left(S N R_{s}=-3 \mathrm{~dB}\right)$.

\section{Simulation Results}

Fig. 4 shows the bias, INPR, ASLR, and PSLR as functions of target density for a simulation with targets with random radar cross section (20-dB dynamic range) and an average $S N R_{s}$ of $17 \mathrm{~dB}$ for the upper panels and $-3 \mathrm{~dB}$ for the lower ones. In all cases, the resampling (or interpolation) factor was set to eight. The results shown in the left panels correspond to cases in which the targets were (unrealistically but conveniently) located on the grid points of the output images, whereas for the results displayed in the right panels, this restriction was not imposed.

Forcing the targets to lay on the grid allows the evaluation of the intrinsic performance of Capon and APES. In this case, it can be seen that the intensity of the targets for the Capon-based estimator is around $2 \mathrm{~dB}$ below that of the rest of the algorithms, while APES is unbiased with respect to the matched filter and the windowed matched filter. When the targets are allowed to lay off the grid, Capon seems to severely underestimate the target amplitude for target densities that are under 0.3 for the high-SNR case. This is caused by most targets being miss-sampled (skipped over) because the effective resolution of Capon is below the grid spacing. The slight increase of bias with target density for the matched filter is caused by the sidelobes of strong targets superposed to weaker ones.

The INPR plots confirm this behavior. As the target density decreases, Capon and APES can adapt more effectively, yielding an improved resolution that, in turn, reduces the INPR. For the DFT and Hamming-DFT cases, the resolution is constant, which results in practically constant INPR. In all four cases, APES-produced images have an INPR that is above the ideal lower bound, which means that no targets are being lost. For the gridded case and high SNR, Capon-generated results for low target densities are a couple of decibels below the ideal lower bound, which is consistent with the observed amplitude bias. When targets are allowed off grid, the INPR drops more than $10 \mathrm{~dB}$ below the ideal lower bound, which is explained, again, by the miss-sampling of a fraction of the targets. Decreasing the SNR results in an increase of INPR or loss of resolution for both Capon and APES. For $S N R_{s}=-3 \mathrm{~dB}$ and target densities approaching zero, there is a trend reversal with a steep increase of INPR. This is because by setting the single-target SNR constant, the aggregate SNR is proportional to the number of targets. As this aggregate SNR decreases, the estimates of the covariance matrices become increasingly diagonal, and the algorithms become less adaptive.

The ASLR and PSLR plots both illustrate sidelobe rejection. As expected, sidelobe rejection is worst for the pure matched filter. Moreover, sidelobe rejection decreases rapidly as the 

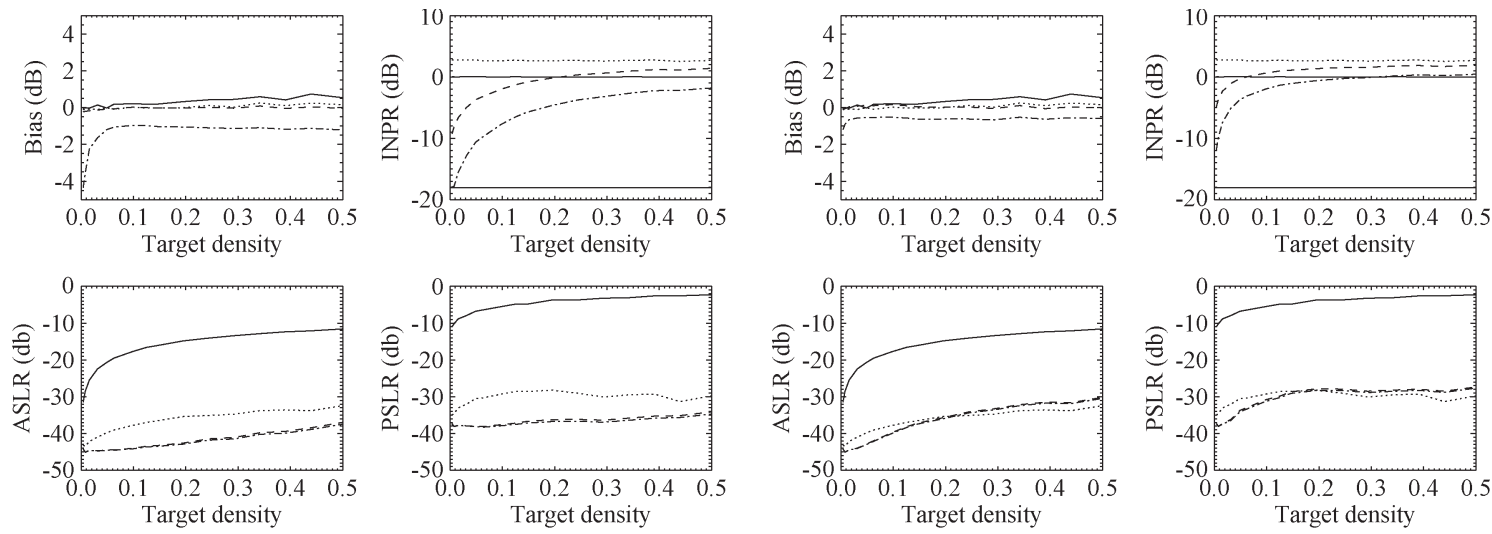

(a)
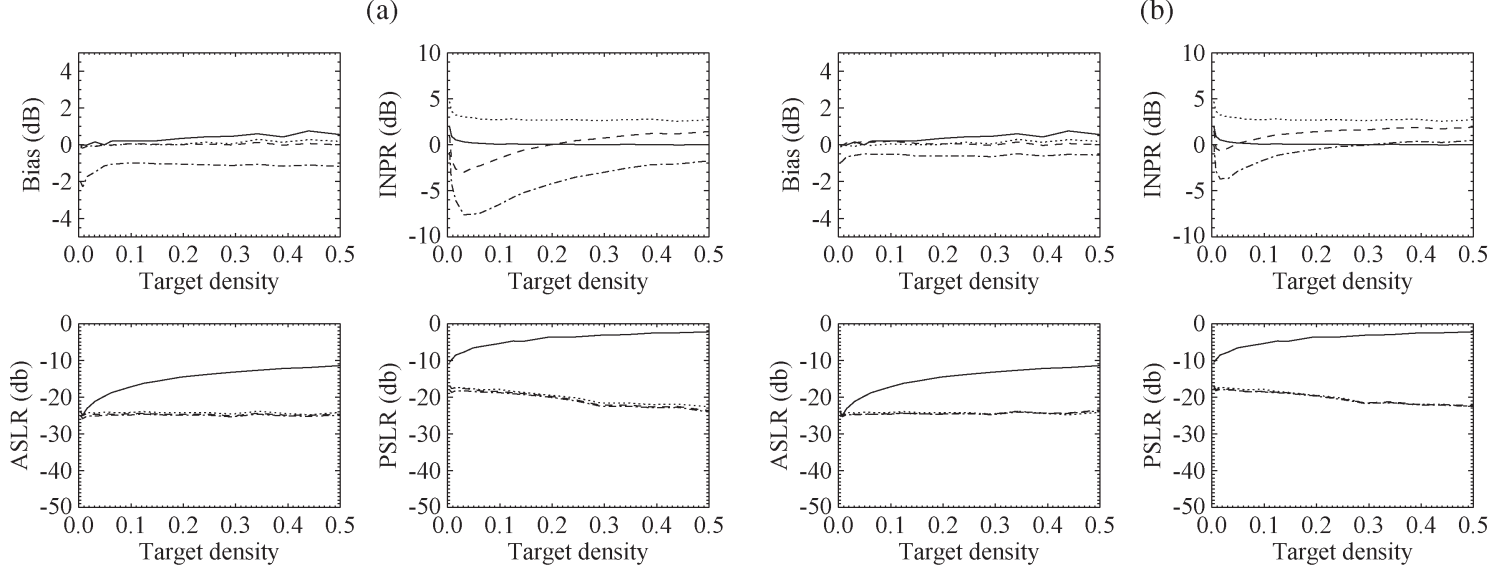

(c)
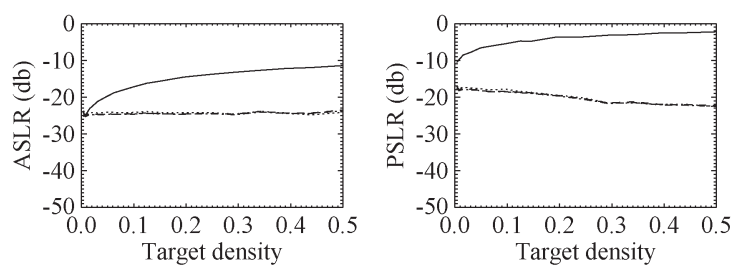

(d)

Fig. 5. Amplitude bias, INPR, ASLR, and PSLR as functions of target density for different combinations of SNR and diagonal loading. See also Fig. 4. (a) $S N R_{\mathrm{dl}}=10 \mathrm{~dB}$ and $S N R_{s}=17 \mathrm{~dB}$. (b) $S N R_{\mathrm{dl}}=0 \mathrm{~dB}$ and $S N R_{s}=17 \mathrm{~dB}$. (c) $S N R_{\mathrm{dl}}=10 \mathrm{~dB}$ and $S N R_{s}=-3 \mathrm{~dB}$. (d) $S N R_{\mathrm{dl}}=0 \mathrm{~dB}$ and $S N R_{s}=-3 \mathrm{~dB}$.

number of targets increases. Also, as expected, Hamming windowing improves both average and peak sidelobe rejections by several orders of magnitude. For high $S N R_{s}$ levels, this rejection still decreases slowly as the target density increases, due to the random addition of multiple sidelobes. Capon and APES yield even lower average and peak sidelobe levels, which tend to be independent of the number of targets. Capon sidelobes are slightly lower, but the difference can be justified by the global downward bias of Capon's power estimates. For low $S N R_{s}$ levels, the calculated sidelobe levels are clearly dominated by noise, resulting in virtually identical ASLR and PSLR for Hamming windowing, APES, and Capon. Thus, the adaptive nature of the APES and Capon estimators does not result in new unwanted artifacts in the presence of noise.

Fig. 4(b) clearly shows the risk of missing targets, even for a high resampling factor, in the case of sparse scenarios with high SNR levels. Clearly, it is desirable to be able to control the required resampling factor. As discussed in Section III-B, this can be accomplished by diagonally loading the covariance matrix, which has an impact on the resolution similar to that of a loss of SNR. Fig. 5 repeats the previous analysis on the same data sets, with the targets off grid, applying two different levels of diagonal loading. By limiting the adaptability of the algorithms, diagonal loading decreases the downward bias of Capon's power estimates and reduces the resolution of APES and Capon. For example, for the high- $S N R_{s}$ case, setting $S N R_{\mathrm{dl}}=10 \mathrm{~dB}$ sets the INPR over the ideal lower bound
( $-18 \mathrm{~dB}$ for $I=8)$, implying that no targets are being missed. At $S N R_{\mathrm{dl}}=0 \mathrm{~dB}$, the minimum INPR is above $-12 \mathrm{~dB}$, which implies that the resampling factor could be lowered to $I=4$. However, for higher target densities, this level of diagonal loading eliminates most or all the resolution gains. Sidelobe levels increase to levels that are very similar to that of the Hamming-windowed matched filter. Diagonal loading in the low-SNR $R_{s}$ case simply yields lower resolution INPR with, in this case, no associated benefit. Therefore, it can be concluded that diagonal loading is an effective tool to mitigate an excess of resolution but that it should be applied in function of the nature of the chip image. The reader should note that in the processing flow proposed, target density and chip-image SNR levels can be determined before applying the Capon or APES algorithm.

Fig. 6 shows a comparison of the rms interferometric phase error for the four different focusing methods. The eight subfigures correspond to the eight cases shown in Figs. 4 and 5. Forcing the targets to lay on the output grid reveals that the intrinsic performance of Capon in terms of its phase error tends to be worse than that of the matched filter or Hammingwindowed matched filter. In contrast, APES behaves consistently better than the nonadaptive methods, except for very low target densities, for which the unwindowed matched filter performs best. Allowing the targets to lay off grid shows how, for Capon, the partial miss-sampling of targets results in very large phase errors. 

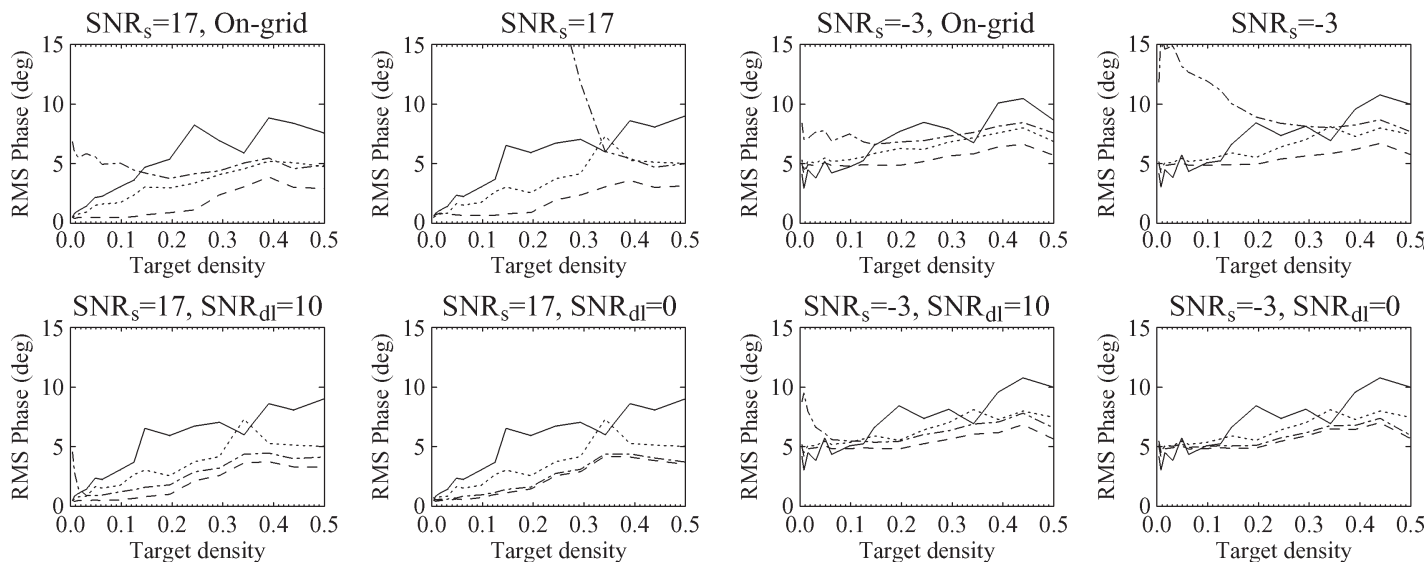

Fig. 6. Interferometric phase error as a function of target density. In all plots, the solid line corresponds to DFT processing (matched filter), the dotted line to DFT after applying a 2-D Hamming window, the dashed line to the APES estimator, and the dash-dot line to Capon processing. Simulation and processing parameters correspond to those of the results shown in Figs. 4 and 5.

Diagonal loading clearly improves the interferometric behavior of Capon beamforming, dropping the rms error below that of the nonadaptive methods but in no case below that of APES. The APES phase error is basically unaffected by diagonal loading. As the SNR drops, all methods produce similar results. It is worth emphasizing that from an interferometric point of view, the APES estimator is much more robust than Capon. This is consistent with the results shown by other authors [16].

\section{Joint Versus Independent Multichannel Processing}

To study the impact of jointly processing several channels in a multichannel scenario, both channels in the simulated interferometric data sets have been focused jointly and independently. Also, the following two scenarios have been considered.

1) In the first scenario, it is assumed that all targets are present in both interferometric channels. Hence, the signal received by both channels is identical except for the added white noise and a random interferometric phase. This case corresponds, for example, to a single-pass interferometric acquisition.

2) In the second scenario, the presence of a number of channel-specific targets (CSTs), i.e., targets that appear in only one of the two channels, was considered. In particular, for the results shown, it was considered that the number of CSTs present in each of the images was onefourth of the number of targets present in both images. These CSTs were assumed to have the same amplitude distribution as the common targets. This second case corresponds can be assimilated to a repeat-pass interferometric or polarimetric case.

Fig. 7 shows some significant results. The left panels in the figure show the amplitude benchmarks for a windowed DFT (dotted lines), APES (dashed lines), and Capon (dashdot), setting the resampling factor $I=8$, random amplitude, and $S N R_{s}=17 \mathrm{~dB}$. To avoid errors due to undersampling, the targets were forced to be located on the output grid. In all plots, the black lines correspond to joint processing, while the gray lines correspond to channel-per-channel processing. The main conclusion is that the only significant difference is that the underestimation of the amplitude by Capon's method is aggravated if the channels are processed one by one. This underestimation is the result of Capon adapting to a particular estimate of the covariance matrix and not the real one (which is unknown). In the case of joint processing, this estimate is obtained using a larger data set and is therefore closer to the true covariance matrix. The small differences in the remaining metrics are also caused by this increased downward bias of the amplitude estimate.

The right panels in Fig. 7 compare the standard deviation of the phase error in high- and low-SNR situations and with or without the presence of CSTs. In the absence of CST and low target densities, Capon improves if the channels are jointly processed. Above a certain target density, however, independent processing produces slightly better results. For APES, there is, as with the amplitude, virtually no difference between joint and independent processing.

Adding an increased number of CSTs degrades the estimated interferometric phase as the entire interferometric pair loses coherence. Other than this general increase of the phase error, for low target densities, the Capon estimator performs better, again, if the channels are jointly processed, while for high target densities, it gives better phase estimates if the channels are processed independently. For APES, the phase errors are consistently lower if the channels are treated one by one. This smaller error for independent processing can easily be understood. For each focused pixel, APES and Capon try to place the minima of the adapted impulse response at the positions of interfering targets. This can be accomplished more successfully if there are less targets to be suppressed. In the case of joint processing, these impulse responses need to suppress simultaneously all interfering targets present in all channels.

The main conclusion of this analysis is that the phase quality is best preserved by using the APES estimator and processing the channels independently. However, the shortcoming of these simulations is that it has only considered collections of point targets. For distributed targets, it may be argued that it is desirable to force all channels to be processed exactly the same way. For example, in many cases, multichannel processing 

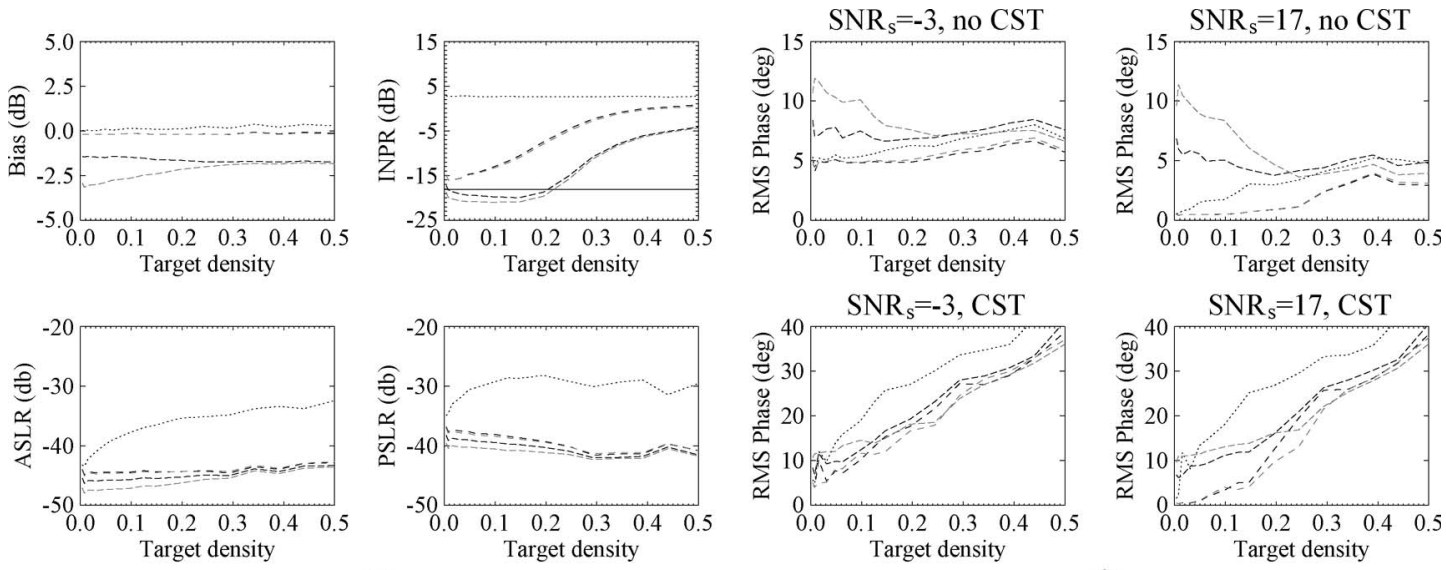

(a)
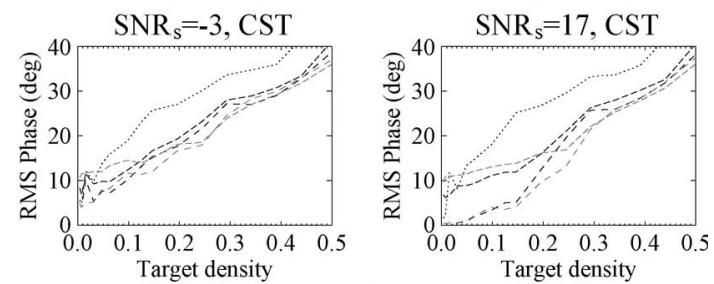

(b)

Fig. 7. Joint versus independent multichannel processing. The left panels show the amplitude benchmarks for the (dotted lines) windowed DFT, (dashed lines) APES, and (dash-dot) Capon, setting the resampling factor $I=8$, random amplitude, and $S N R_{s}=17 \mathrm{~dB}$ and forcing all targets to be located on the output grid. In all plots, the black lines correspond to joint processing of both interferometric channels, while the gray lines correspond to independent processing of each channel. The right panels compare the phase errors of Capon and APES by applying joint and independent processing. In the upper channels, no CSTs have been considered, while in the lower panels, a fraction of the targets were specific to one of the two channels.

requires that the spatial resolution is constant across channels. This can only be guaranteed by jointly processing all channels.

\section{EXPERIMENTAL RESULTS}

The algorithms discussed in the previous sections have been applied to data sets generated by the European Space Agency's ERS-1, ERS-2, and ENVISAT missions and Canada's RADARSAT-2. In particular, the results shown in this section correspond to a RADARSAT-2 fine quad-polarization-mode data set acquired on November 28, 2008, in a descending pass over Barcelona (Spain). This data set has been selected because it allows the qualitative evaluation of the multichannel behavior of Capon and APES without having to deal with, for example, image coregistration, temporal decorrelation, or Doppler centroid offsets between images.

The data were reprocessed using the provided single look complex (SLC) data as an input. In order to equalize the data, the azimuth spectrum, which results from the combination of antenna azimuth beam pattern and the Kaiser windowing applied by the SAR processor, was estimated from the data. The range spectrum was generated using the chirp replica and Kaiser window parameters provided with the SLC data. In both cases, portions of the range and azimuth spectra that were more than $6 \mathrm{~dB}$ below the power level at the center of the spectra were eliminated. In azimuth, this results in a processed Doppler bandwidth of approximately $692 \mathrm{~Hz}$ (down from the $855-\mathrm{Hz}$ total Doppler bandwidth processed in the original SLC) and a processed pulse bandwidth of $26.07 \mathrm{MHz}$ (down from $30 \mathrm{MHz}$ in the original SLC). The data have been processed using chipimage sizes of $32 \times 32$ and $48 \times 48$, which produce practically indistinguishable results.

The four polarimetric channels have been processed jointly and one by one. For APES, this produced very similar results, which is consistent with the simulations. For Capon, one-byone processing resulted in better resolved targets but a clear downward bias of the amplitude. In contrast, if the channels were jointly processed, the results produced by the Capon estimator look qualitatively very similar to the ones generated by APES. The results that will be discussed hereinafter correspond to channel-by-channel processing.

Fig. 8 shows an RGB composite HH-channel reflectivity image of a portion of the acquired data set showing most of downtown Barcelona. The RGB channels correspond to the original SLC (red), and the image was reprocessed using APES (green) and Capon (blue). The RGB composite has been generated so that the red, green, and blue reflect the relative amplitudes of the original SLC, APES, and Capon, while the intensity of the image is proportional to the power of each pixel, averaged over the three images, and expressed in decibels. Samples and lines refer to the original data, although the APES and Capon data have been resampled by a factor of approximately three in range and two in azimuth. The minus sign in front of the line number indicates that the image has been reversed in azimuth. In the RGB composite, pixels that are equal in the three images show up as gray levels, while any departure from gray reveals differences between the images. In particular, the reddish regions in the figure correspond mainly to the sidelobes in the original SLC that have been suppressed by APES and Capon. The downward bias of Capon, which attenuates the blue channel with respect to the red and green ones, gives the entire image a brownish tone. There are a number of artifacts in the part of the image corresponding to the sea, which are aligned in azimuth to bright targets on land. Similar artifacts are present in other RADARSAT-2 images corresponding to coastal areas. They may be related to saturation effects caused by land targets when the automatic gain control adjusts the receiver gain to the low reflectivity of the sea. The amplitude of these artifacts is neither reduced nor increased by Capon or APES processing with respect to the original SLC image.

Fig. 9 shows a comparison of the original data with APESand Capon-reprocessed images for a detail of Barcelona. The upper right panel shows an orthophotograph of the detail, rotated to match the orientation of the SAR acquisition. The upper left panel shows an RGB composite (red: original, green: APES, and blue: Capon) of the VV-channel reflectivity for the selected region. The remaining left panels show separately HH reflectivity images of the original SLC, APES, 


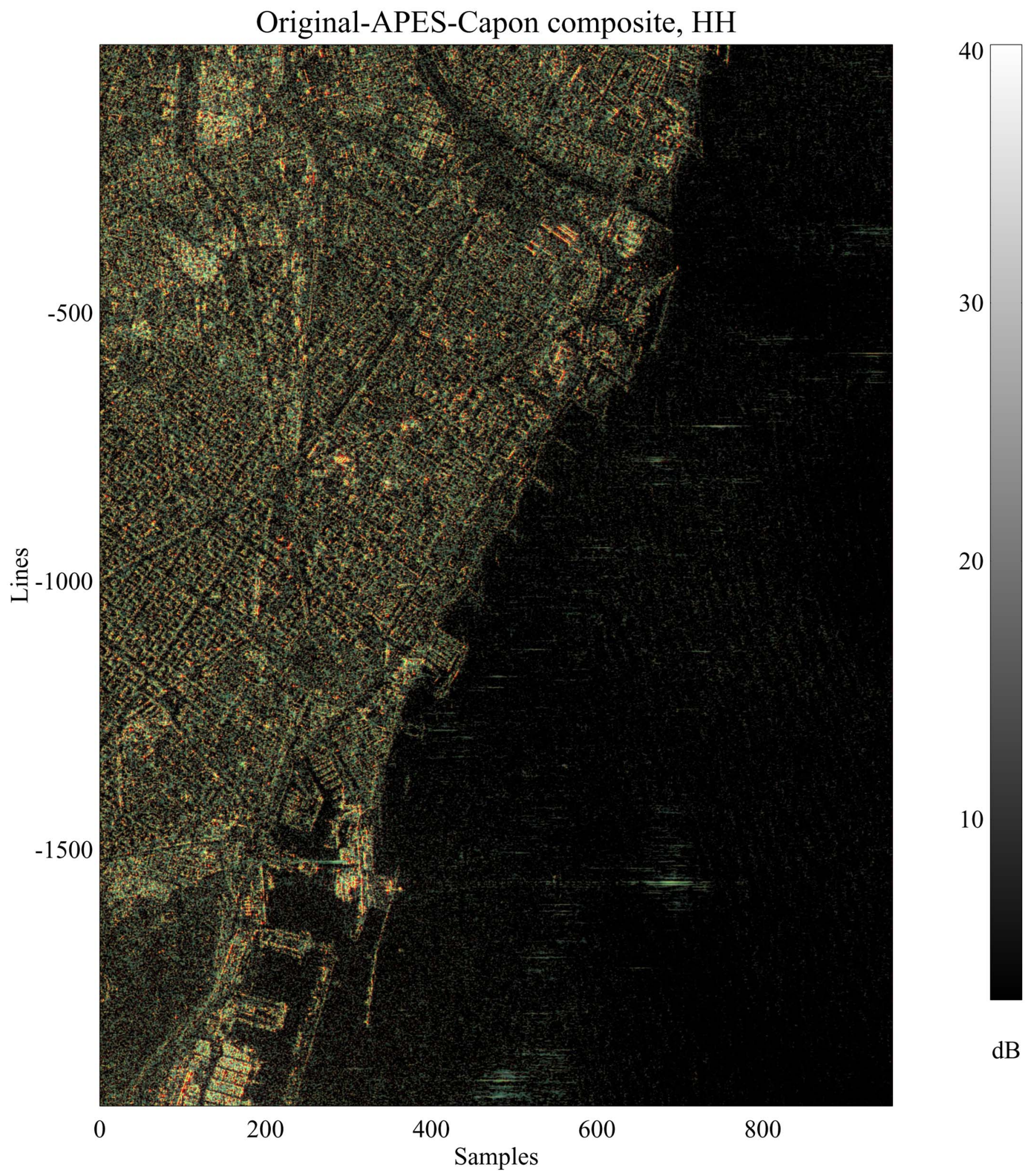

Fig. 8. RGB composite showing a RADARSAT-2 reflectivity image corresponding to Barcelona (Spain). The RGB channels correspond to (red) the original SLC, and the image was reprocessed using (green) APES and (blue) Capon. RADARSAT-2 was operated in fine-resolution full-polarimetric mode. The data shown correspond to the $\mathrm{HH}$ channel. The samples and lines refer to the original data.

and Capon. The most remarkable feature is the suppression by APES and Capon of the sidelobes of the bright target at position $(170,-110)$. Also, in general, targets appear better resolved in the APES and Capon images than in the original one. This combination of sidelobe reduction and improved resolution is highlighted by the red regions surrounding some targets in the composite image. With respect to APES, the amplitudes obtained by the Capon estimator are clearly biased 

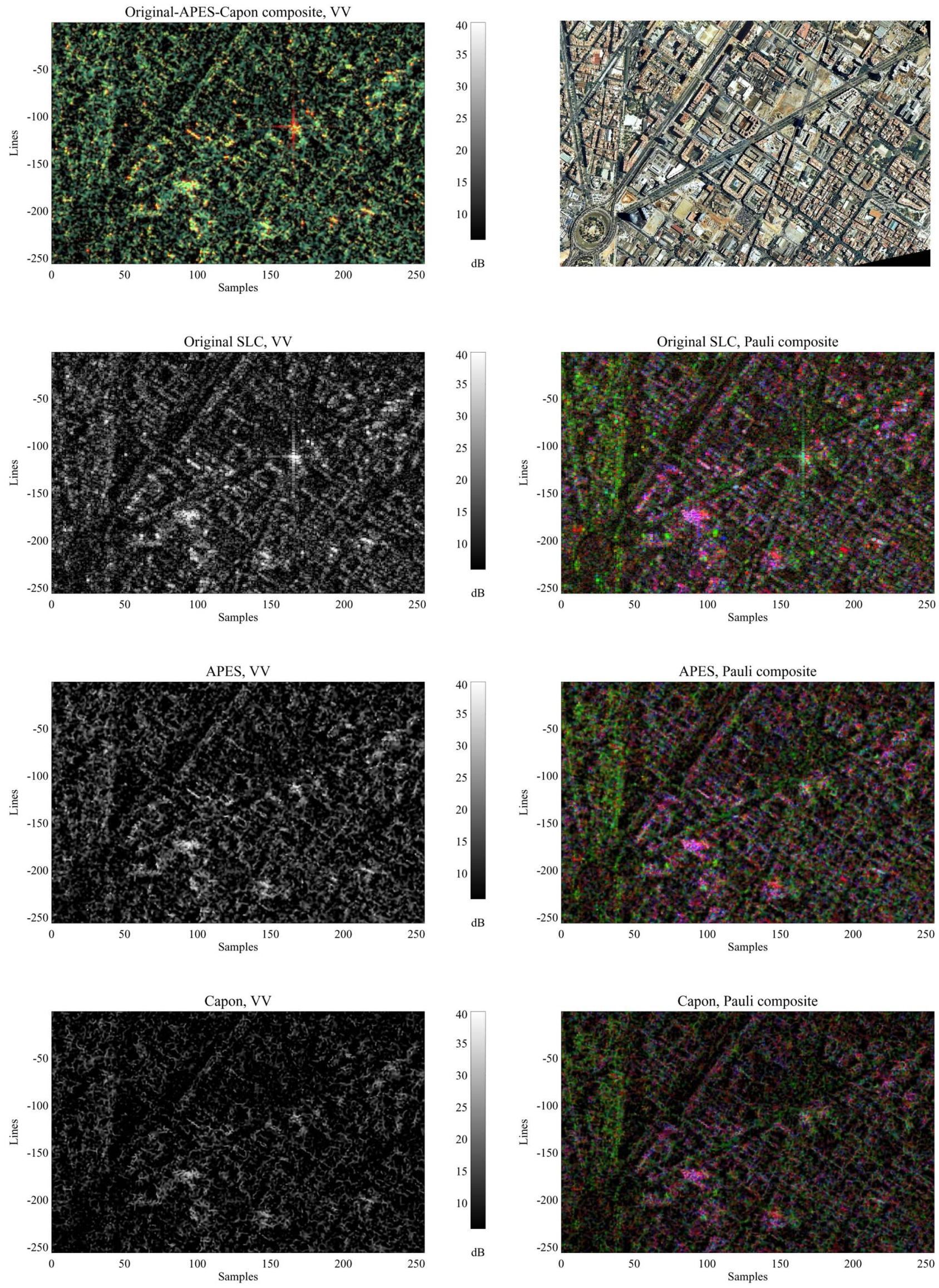

Fig. 9. Comparison of the original SLC with APES and Capon reprocessed images for a detail of Barcelona. 

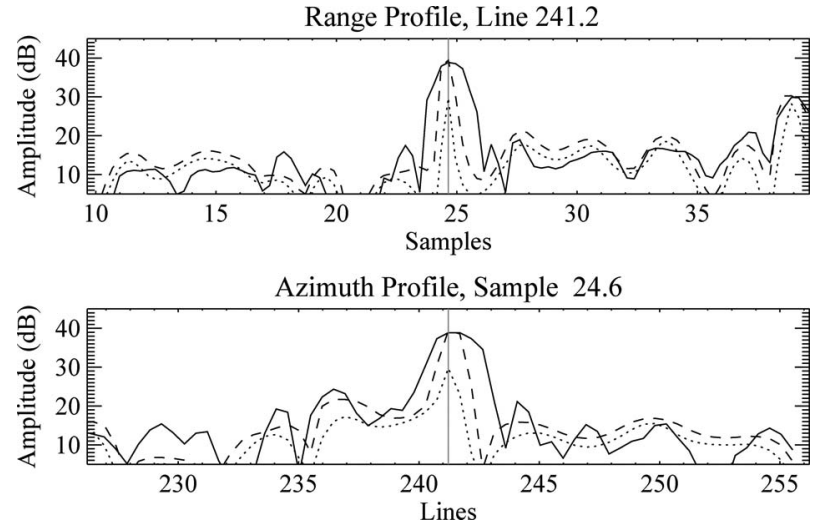

Fig. 10. Comparison of range and azimuth profiles of target of opportunity. The solid lines show the original data interpolated by applying zero padding in the frequency domain. The dashed and dotted lines correspond to the APESand Capon-reprocessed images, respectively. The samples and lines are with respect to the results shown in Fig. 9.

downward, while the features in the image appear better resolved.

Fig. 10 shows a comparison of range and azimuth profiles of target of opportunity located at sample 24.6 and line 241.2 with respect to the images shown in Fig. 9. The solid lines show the original data interpolated by applying zero padding in the frequency domain. The dashed and dotted lines correspond to the APES- and Capon-reprocessed images, respectively. This example illustrates the resolution gain of both algorithms. The underestimation of the amplitude by Capon's MVM estimator is also clearly shown.

The lower three right panels in Fig. 9 show an RGB composite of the Pauli decomposition [20] of the four polarimetric channels for the original data, APES, and Capon. The red, green, and blue channels correspond to $\mathrm{HH}+\mathrm{VV}$ (odd-bounce), $\mathrm{VV}-\mathrm{HH}$ (even-bounce), and HV+HV. For example, the green region at the left of the images corresponds to an area where the buildings are well aligned with the along-track direction, which results in a large number of properly oriented dihedral (double-bounce) scatterers. The Pauli signature of the scatterers is mostly preserved in the three images. Because a consistent estimation of odd- and even-bounce Pauli channels requires that the relative phase of $\mathrm{HH}$ and $\mathrm{VV}$ is preserved, these results illustrate qualitatively that the phase of the reprocessed images has been preserved.

\section{CONCLUSION}

An implementation of a SAR processing chain based on Capon's MVM estimator and APES has been presented, discussing several of the parameters that need to be considered. This processing chain can use already focused SLC data, which simplifies its implementation and allows the reprocessing of small regions of interest. It should be highlighted that the spectra SLC images fed to the algorithms need to be equalized to remove any tapering introduced explicitly during processing or implicitly by the antenna beam pattern.

For multichannel scenarios, the option of processing each channel independently has been compared to a joint-processing approach, for which the efficient implementation of APES proposed in [9] has been generalized to a multichannel case. For APES, we have found no benefit in using this joint-processing approach, particularly in the common case that there are targets that are not present in all channels. For Capon, this jointprocessing approach seems to stabilize the algorithm but at the cost of reducing its resolution.

Numerical simulations and a qualitative analysis of results using real RADARSAT-2 data confirm that, as reported by other authors, APES is unbiased while Capon tends to underestimate the radar brightness. We have found also that the phase is better preserved using APES. These larger phase and amplitude errors in the case of Capon are a result of an overadaptation to the data. This overadaptation can be mitigated by applying some level of diagonal loading to find a compromise between resolution gain and amplitude and phase errors.

As it is often the case in engineering, there is no simple answer to the question of which algorithm is better. APES is numerically more stable than Capon's MVM and is radiometrically more precise. For moderate target densities, its resolution is only slightly better than the matched-filter resolution but with superior sidelobe rejection. It may therefore be a good choice for applications in which the radiometric performance is critical or in interferometric applications. However, it must be noted that for many applications that require good radiometric performance, the conventional tapered matched filter may be the most suitable option.

In terms of resolution, Capon is the superior method. It has the appealing property that this resolution, even for fairly high target densities, improves as the SNR increases. For applications in which target discrimination is critical, it may be the preferred choice. In particular, in a polarimetric case, Capon may be able to discriminate close targets with different polarimetric signature where APES or a traditional matchedfilter approach cannot. As shown in the Appendix, Capon processing is significantly simpler than APES and therefore requires significantly less computing resources. In fact, Capon estimates can be computed with almost no added cost as a byproduct of the APES algorithm.

Future work will focus on testing and applying APES and Capon to InSAR, DInSAR, and PolDInSAR. Using large data sets in DInSAR should allow a real-life evaluation of the phase quality of both algorithms. Also, DInSAR in urban areas, where sidelobe mitigation is a real issue and large sets of SLCs are available, can be expected to benefit directly from these algorithms.

Another possible research direction is to find criteria and algorithms to automatically select some of the processing parameters. For example, for Capon-processed images, it would be interesting to be able to estimate the required grid spacing to avoid the miss-sampling of targets.

\section{APPENDIX \\ CAPON AND APES IMPLEMENTATION}

The Capon and APES estimators used in this paper have been implemented following the efficient algorithm proposed in [9]. However, this derivation had to be extended to allow interferometric processing. Because it is desirable that the two images used to generate an interferogram are processed in exactly the same way, the adaptive filter used needs to be generated using both data sets simultaneously. This appendix discusses the modifications of the original implementation required for 
this interferometric coprocessing. The reader is referred to [9] for an in-depth discussion of the algorithm and to [16] for a theoretical discussion of an interferometric extension to the APES algorithm.

For an $N_{1} \times N_{2}$ data block with elements $z_{n_{1}, n_{2}}$, both estimators construct first a set of $L_{1} \times L_{2}$ overlapping $M_{1} \times M_{2}$ subimages $\mathbf{Z}_{\mathbf{l}_{1}, \mathbf{l}_{2}}$ with elements $z_{l_{1}, l_{2}, n_{1}, n_{2}}=z_{l_{1}+n_{1}, l_{2}+n_{2}}$ and a set of reversed and conjugated subimages $\tilde{\mathbf{Z}}_{1_{1}, \mathbf{l}_{2}}$ with elements $\tilde{z}_{l_{1}, l_{2}, n_{1}, n_{2}}=z_{N_{1}-1-l_{1}-n_{1}, N_{2}-1-l_{2}-n_{2}}^{*}$. These subimages can be represented as vectors $\tilde{\mathbf{z}}_{1_{1}, \mathbf{l}_{2}}$ and $\tilde{\mathbf{z}}_{1_{1}, \mathbf{l}_{2}}$ by stacking their respective columns. It will also be useful to consider the matrix

$$
\mathbf{Z}_{1}=\left[\begin{array}{llll}
\mathbf{z}_{0,0} & \mathbf{z}_{1,0} & \cdots & \mathbf{z}_{\mathbf{L}_{1}-1, \mathbf{L}_{2}-1}
\end{array}\right]
$$

that contains all the vectorized overlapping subimages, the $M_{1}$. $M_{2}$ element vector resulting from calculating the 2-D DFT of the series $\mathbf{z}_{\mathbf{l}_{1}, \mathbf{l}_{2}}$

$$
\mathbf{g}_{\mathbf{1}}\left(\omega_{1}, \omega_{2}\right)=\sum_{l_{1}=0}^{L_{1}-1} \sum_{l_{2}=0}^{L_{2}-1} \mathbf{z}_{\mathbf{l}_{1}, \mathbf{l}_{2}} e^{-j\left(\omega_{1} l_{1}+\omega_{2} l_{2}\right)}
$$

and $\tilde{\mathbf{g}}_{1}\left(\omega_{1}, \omega_{2}\right)$, which is computed likewise from $\tilde{\mathbf{z}}_{\mathbf{l}_{1}, \mathbf{l}_{2}}$.

For a single image, the forward-backward estimate of the covariance matrix needed by Capon and APES is given by

$$
\hat{\mathbf{R}}_{1}=\sum_{l_{1}=0}^{L_{1}-1} \sum_{l_{2}=0}^{L_{2}-1}\left(\mathbf{z}_{\mathbf{l}_{1}, \mathbf{l}_{\mathbf{2}}} \mathbf{z}_{\mathbf{l}_{1}, \mathbf{l}_{\mathbf{2}}}^{\mathbf{H}}+\tilde{\mathbf{z}}_{\mathbf{l}_{1}, \mathbf{l}_{\mathbf{2}}} \tilde{\mathbf{z}}_{\mathbf{l}_{1}, \mathbf{l}_{\mathbf{2}}}^{\mathbf{H}}\right) .
$$

The single-image Capon estimator can then be expressed as

$$
\alpha\left(\omega_{1}, \omega_{2}\right)=\frac{\mathbf{a}_{M}^{H} \hat{\mathbf{R}}_{1}^{-1} \mathbf{g}_{1}}{L_{1} L_{2} \mathbf{a}_{M}^{H} \hat{\mathbf{R}}_{1}^{-1} \mathbf{a}_{M}} .
$$

In order to use Capon's estimator, if a time series of two or more images is available, it is sufficient to use (19) to estimate the covariance matrix $\left(\hat{\mathbf{R}}_{k}\right)$ for each image $k$ and average those to produce a joint estimation

$$
\hat{\mathbf{R}}=\frac{1}{K} \sum_{k=0}^{K-1} \hat{\mathbf{R}}_{k}
$$

which can be plugged into the algorithm. The Capon estimator is then given by

$$
\alpha\left(\omega_{1}, \omega_{2}\right)=\frac{\mathbf{a}_{M}^{H} \hat{\mathbf{R}}^{-1} \mathbf{G}}{L_{1} L_{2} \mathbf{a}_{M}^{H} \hat{\mathbf{R}}^{-1} \mathbf{a}_{M}}
$$

where

$$
\mathbf{G}\left(\omega_{1}, \omega_{2}\right)=\left[\mathbf{g}_{\mathbf{0}}\left(\omega_{1}, \omega_{2}\right), \ldots, \mathbf{g}_{\mathbf{K}-\mathbf{1}}\left(\omega_{1}, \omega_{2}\right)\right]
$$

is the matrix whose columns are the vectors $\tilde{\mathbf{g}}_{\mathbf{k}}\left(\omega_{1}, \omega_{2}\right)$ and $\tilde{\mathbf{G}}\left(\omega_{1}, \omega_{2}\right)$ is constructed likewise from the vectors $\tilde{\mathbf{g}}_{\mathbf{k}}\left(\omega_{1}, \omega_{2}\right)$.

The single-image APES estimator requires the use of an expression that is identical to (20), replacing the estimate of covariance matrix $\hat{\mathbf{R}}$ by an estimate of

$$
\begin{aligned}
\hat{\mathbf{Q}}_{1}\left(\omega_{1}, \omega_{2}\right)=\hat{\mathbf{R}}_{1}- & {\left[\mathbf{g}_{\mathbf{1}}\left(\omega_{1}, \omega_{2}\right) \mathbf{g}_{\mathbf{1}}^{H}\left(\omega_{1}, \omega_{2}\right)\right.} \\
+ & \left.\tilde{\mathbf{g}}_{\mathbf{1}}\left(\omega_{1}, \omega_{2}\right) \tilde{\mathbf{g}}_{\mathbf{1}}^{H}\left(\omega_{1}, \omega_{2}\right)\right] /\left(L_{1} L_{2}\right) .
\end{aligned}
$$

The APES estimator in an interferometric context is analyzed in [16]. The interferometric version of matrix $\hat{\mathbf{Q}}$ found there can be written as

$$
\begin{aligned}
\hat{\mathbf{Q}}\left(\omega_{1}, \omega_{2}\right)=\hat{\mathbf{R}} & -\mathbf{G}\left(\omega_{1}, \omega_{2}\right)\left[\frac{1}{K L_{1} L_{2}} \mathbf{I}_{\mathbf{K}}\right] \mathbf{G}^{H}\left(\omega_{1}, \omega_{2}\right) \\
& +\tilde{\mathbf{G}}\left(\omega_{1}, \omega_{2}\right)\left[\frac{1}{K L_{1} L_{2}} \mathbf{I}_{\mathbf{K}}\right] \tilde{\mathbf{G}}^{H}\left(\omega_{1}, \omega_{2}\right)
\end{aligned}
$$

where $\mathbf{I}_{\mathbf{K}}$ represents the $K \times K$ identity matrix and $K$ is the number of images being jointly processed. Using the matrix inversion lemma and dropping the frequency arguments, the inverse of (23) can be written as

$$
\hat{\mathbf{Q}}^{-1}=\tilde{\mathbf{Q}}^{-1}+\tilde{\mathbf{Q}}^{-1} \mathbf{G} \mathbf{P}_{\tilde{\mathbf{Q}}}^{-1} \mathbf{G}^{H} \tilde{\mathbf{Q}}^{-1}
$$

with

$$
\tilde{\mathbf{Q}}^{-1}=\hat{\mathbf{R}}^{-1}+\hat{\mathbf{R}}^{-1} \tilde{\mathbf{G}} \mathbf{P}_{\hat{\mathbf{R}}}^{-1} \tilde{\mathbf{G}}^{H} \hat{\mathbf{R}}^{-1}
$$

where, for notational convenience, we have defined the $K \times K$ matrices

$$
\begin{aligned}
& \mathbf{P}_{\tilde{\mathbf{Q}}}=K L_{1} L_{2} \mathbf{I}_{\mathbf{K}}-\mathbf{G}^{H} \tilde{\mathbf{Q}}^{-1} \mathbf{G} \\
& \mathbf{P}_{\hat{\mathbf{R}}}=K L_{1} L_{2} \mathbf{I}_{\mathbf{K}}-\tilde{\mathbf{G}}^{H} \hat{\mathbf{R}}^{-1} \tilde{\mathbf{G}} .
\end{aligned}
$$

The APES estimator can be written as

$$
\begin{aligned}
\alpha\left(\omega_{1}, \omega_{2}\right) & =\frac{\mathbf{a}_{M}^{H} \hat{\mathbf{Q}} \mathbf{G}}{L \mathbf{a}_{M}^{H} \hat{\mathbf{Q}}^{-1} \mathbf{a}_{M}} \\
& =\frac{K L_{1} L_{2} \mathbf{a}_{M}^{H} \tilde{\mathbf{Q}} \mathbf{G} \mathbf{P}_{\tilde{\mathbf{Q}}}^{-1}}{\mathbf{a}_{M}^{H} \tilde{\mathbf{Q}}^{-1} \mathbf{a}_{M}+\mathbf{a}_{M}^{H} \tilde{\mathbf{Q}}^{-1} \mathbf{G} \mathbf{P}_{\tilde{\mathbf{Q}}}^{-1} \mathbf{G}^{H} \tilde{\mathbf{Q}}^{-1} \mathbf{a}_{M}}
\end{aligned}
$$

where the $M=M_{1} \cdot M_{2}$ element vector $\mathbf{a}_{M}$ holds the coefficients to calculate the 2-D DFT for frequency $\left(\omega_{1}, \omega_{2}\right)$. The efficient implementation of APES derived in [9] uses the Cholesky factorization of the estimated covariance matrix, such that $\hat{\mathbf{R}}^{-1}=\hat{\mathbf{C}}^{-1}\left(\hat{\mathbf{C}}^{-1}\right)^{H}$, and defines the $M \times 1$ vector

$$
\mathbf{b}=\mathbf{a}_{M}^{H} \hat{\mathbf{C}}^{-1}
$$

and the $M \times K$ matrices

$$
\begin{aligned}
& \mathbf{D}=\left[\begin{array}{lll}
\mathbf{D}_{\mathbf{0}} \mathbf{a}_{L}^{*} & \cdots & \mathbf{D}_{\mathbf{K}-\mathbf{1}} \mathbf{a}_{L}^{*}
\end{array}\right] \\
& \mathbf{E}=\left[\begin{array}{lll}
\mathbf{E}_{\mathbf{0}} \mathbf{a}_{L}^{*} & \cdots & \mathbf{E}_{\mathbf{K}-\mathbf{1}} \mathbf{a}_{L}^{*}
\end{array}\right]
\end{aligned}
$$

where $\mathbf{D}_{\mathbf{i}}=\left(\hat{\mathbf{C}}^{-1}\right)^{H} \mathbf{Z}_{\mathbf{i}}$ and $\mathbf{E}_{\mathbf{i}}=\left(\hat{\mathbf{C}}^{-1}\right)^{H} \tilde{\mathbf{Z}}_{\mathbf{i}}$. These expressions can be evaluated simultaneously for all desired 
frequencies using FFT algorithms. Now, several of the terms in (29) can be written and efficiently calculated as

$$
\begin{aligned}
\mathbf{a}_{M}^{H} \tilde{\mathbf{Q}}^{-1} \mathbf{G} & =\mathbf{b}^{t} \mathbf{D}+\mathbf{b}^{t} \mathbf{E} \mathbf{P}_{\hat{\mathbf{R}}}^{-1} \mathbf{E}^{H} \mathbf{D} \\
\mathbf{G}^{H} \tilde{\mathbf{Q}}^{-1} \mathbf{G} & =\mathbf{D}^{H} \mathbf{D}+\mathbf{D}^{H} \mathbf{E} \mathbf{P}_{\hat{\mathbf{R}}}^{-1} \mathbf{E}^{H} \mathbf{D} \\
\mathbf{a}_{M}^{H} \tilde{\mathbf{Q}}^{-1} \mathbf{a}_{M} & =\|\mathbf{b}\|^{2}+\mathbf{b}^{t} \mathbf{E} \mathbf{P}_{\hat{\mathbf{R}}}^{-1} \mathbf{E}^{H} \mathbf{b}^{*}
\end{aligned}
$$

The Capon estimator defined in (22) can also be expressed as

$$
\alpha\left(\omega_{1}, \omega_{2}\right)=\frac{\mathbf{b}^{t} \mathbf{D}}{L_{1} L_{2}\|\mathbf{b}\|^{2}}
$$

which can also be computed efficiently. Note that most of the computations required to implement the Capon estimator are also required to implement the APES estimator. Therefore, the Capon estimate can be obtained as a by-product of APES.

\section{ACKNOWLEDGMENT}

The RADARSAT-2 data were provided by MacDonald, Dettwiler and Associates Ltd. under the SOAR 3084 project.

\section{REFERENCES}

[1] C. Cafforio, C. Prati, and F. Rocca, "SAR data focusing using seismic migration techniques," IEEE Trans. Aerosp. Electron. Syst., vol. 27, no. 2, pp. 194-207, Mar. 1991.

[2] R. K. Raney, H. Runge, R. Bamler, I. Cumming, and F. H. Wong, "Precision SAR processing using chirp scaling," IEEE Trans. Geosci. Remote Sens., vol. 32, no. 4, pp. 786-799, Jul. 1994.

[3] A. Moreira, J. Mittermayer, and R. Scheiber, "Extended chirp scaling algorithm for air- and spaceborne SAR data processing in stripmap and ScanSAR imaging modes," IEEE Trans. Geosci. Remote Sens., vol. 34, no. 5, pp. 1123-1136, Sep. 1996.

[4] R. Bamler, "A comparison of range-Doppler and wavenumber domain SAR focusing algorithms," IEEE Trans. Geosci. Remote Sens., vol. 30, no. 4, pp. 706-713, Jul. 1992.

[5] S. R. DeGraaf, "SAR imaging via modern 2-D spectral estimation methods," IEEE Trans. Image Process., vol. 7, no. 5, pp. 729-761, May 1998.

[6] J. Capon, "High-resolution frequency-wavenumber spectrum analysis," Proc. IEEE, vol. 57, no. 8, pp. 1408-1418, Aug. 1969.

[7] P. Stoica, H. Li, and J. Li, "A new derivation of the APES filter," IEEE Signal Process. Lett., vol. 6, no. 8, pp. 205-206, Aug. 1999.

[8] J. Li and P. Stoica, "An adaptive filtering approach to spectral estimation and SAR imaging," IEEE Trans. Signal Process., vol. 44, no. 6, pp. $1469-$ 1484, Jun. 1996.

[9] Z. S. Liu, H. Li, and J. Li, "Efficient implementation of Capon and APES for spectral estimation," IEEE Trans. Aerosp. Electron. Syst., vol. 34, no. 4, pp. 1314-1319, Oct. 1998.

[10] R. D. Palmer, S. Gopalam, T. Yu, and S. Fukao, "Coherent radar imaging using Capon's method," Radio Sci., vol. 33, no. 6, pp. 1585-1598, 1998.

[11] F. Lombardini and A. Reigber, "Adaptive spectral estimation for multibaseline SAR tomography with airborne L-band data," in Proc. IEEE IGARSS, Jul. 2003, vol. 3, pp. 2014-2016.

[12] H. Krim and M. Viberg, "Two decades of array signal processing research: The parametric approach," IEEE Signal Process. Mag., vol. 13, no. 4, pp. 67-94, Jul. 1996.

[13] C. D. Richmond, "Capon algorithm mean-squared error threshold SNR prediction and probability of resolution," IEEE Trans. Signal Process., vol. 53, no. 8, pp. 2748-2764, Aug. 2005.

[14] C. D. Richmond, "On the probability of resolution for the amplitude and phase estimation (APES) spectral estimator," in Proc. ICASSP, 2005, pp. iv/1025-iv/1028.
[15] G. Amdahl, "Validity of the single processor approach to achieving large-scale computing capabilities," in Proc. AFIPS Conf., 1967, vol. 30, pp. 483-485.

[16] M. R. Palsetia and J. Li, "Using APES for interferometric SAR imaging," IEEE Trans. Image Process., vol. 7, no. 9, pp. 1340-1353, Sep. 1998.

[17] B. D. Carlson, "Covariance matrix estimation errors and diagonal loading in adaptive arrays," IEEE Trans. Aerosp. Electron. Syst., vol. 24, no. 4, pp. 297-401, Jul. 1998.

[18] J. Li, P. Stoica, and Z. Wang, "On robust Capon beamforming and diagonal loading," IEEE Trans. Signal Process., vol. 51, no. 7, pp. 1702-1715, Jul. 2003.

[19] C. D. Richmond, R. R. Nadakuditi, and A. Edelman, "Asymptotic mean square error performance of diagonally loaded Capon-MVDR processor," in Proc. Asilomar Conf. Signals, Syst., Comput., Pacific Grove, CA, 2005, pp. 1711-1716.

[20] S. R. Cloude and E. Pottier, "A review of target decomposition theorems in radar polarimetry," IEEE Trans. Geosci. Remote Sens., vol. 34, no. 2, pp. 498-518, Mar. 1996.

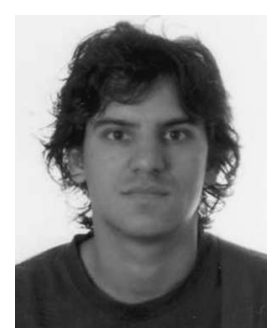

Paco (Francisco) López-Dekker (S'98-M'03) was born in Nijmegen, The Netherlands, in 1972. He received the Ingeniero degree in telecommunication engineering from the Universitat Politècnica de Catalunya (UPC), Barcelona, Spain, in 1997, the M.S. degree in electrical and computer engineering from the University of California, Irvine, in 1998, and the Ph.D. degree from the University of Massachusetts (UMass), Amherst, in 2003, researching on clear-air imaging radar systems to study the atmospheric boundary layer.

In 1999, he joined the Microwave Remote Sensing Laboratory, UMass, as a Ph.D. student. In 2003, he returned to Barcelona, to work for Starlab, a privately held company, to work on the development of GNSS-R sensors, and in 2004, he joined the Department of Telecommunications and Systems Engineering, Universitat Autonoma de Barcelona, Bellaterra, Spain, as a Visiting Professor. Since November 2009, he has been with the Microwaves and Radar Institute, German Aerospace Center (DLR), Oberpfaffenhofen, Germany. His current research is focused on the study of future SAR missions and novel mission concepts.

Dr. López-Dekker was awarded a five-year Ramon y Cajal grant to conduct research on bistatic SAR at the Remote Sensing Laboratory, UPC, in 2005, wherein he joined in March 2006, and the Balsells Fellowship for his M.S. studies at the University of California, Irvine. At the university he has taught courses on signals and systems, signal processing, communication systems, radiation, and guided waves.

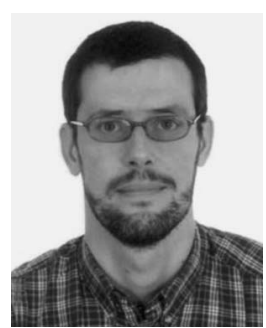

Jordi J. Mallorquí (S'93-M'96) was born in Tarragona, Spain, in 1966. He received the Ingeniero degree in telecommunication engineering and the Dr. Ing. degree in telecommunications engineering from the Universitat Politècnica de Catalunya (UPC), Barcelona, Spain, in 1990 and 1995, respectively, researching on microwave tomography for biomedical applications.

In 1991, he joined the Department of Signal Theory and Communications, UPC, as a Ph.D. student. In 1993, he became an Assistant Professor, and since 1997, he has been an Associate Professor at the Telecommunications Engineering School, UPC. His teaching activity involves microwaves, radionavigation systems, and remote sensing. He spent a sabbatical year at the Jet Propulsion Laboratory, Pasadena, CA, in 1999, working on interferometric airborne SAR calibration algorithms. He is currently working on the application of SAR interferometry to terrain deformation monitoring with orbital and airborne data, vessel detection and classification from SAR images, and 3-D electromagnetic simulation of SAR systems. He is also collaborating in the design and construction of a ground-based SAR interferometer for landslide control. Finally, he is currently developing the hardware and software of a bistatic parasitic SAR for interferometric applications using ERS and ENVISAT as sensors of opportunity. He has published more than 80 papers on microwave tomography, electromagnetic numerical simulation, and SAR processing, interferometry, and differential interferometry in referred journals and international symposia. 